\title{
Short-term memory and the attentional blink: Capacity versus content
}

\author{
ELKAN G. AKYÜREK and BERNHARD HOMMEL \\ Leiden University, Leiden, The Netherlands
}

\begin{abstract}
When people monitor the rapid serial visual presentation (RSVP) of stimuli for two targets (T1 and T2), they often miss T2 if it falls into a time window of about half a second after T1 onset, a phenomenon known as the attentional blink (AB). We found that overall performance in an RSVP task was impaired by a concurrent short-term memory (STM) task and, furthermore, that this effect increased when STM load was higher and when its content was more task relevant. Loading visually defined stimuli and adding articulatory suppression further impaired performance on the RSVP task, but the size of the $\mathrm{AB}$ over time (i.e., T1-T2 lag) remained unaffected by load or content. This suggested that at least part of the performance in an RSVP task reflects interference between competing codes within STM, as interference models have held, whereas the $\mathrm{AB}$ proper reflects capacity limitations in the transfer to STM, as consolidation models have claimed.
\end{abstract}

Human attention is limited with respect to space, a broadly investigated dimension, and with respect to time, as demonstrated in tasks with a rapid serial visual presentation (RSVP) of stimulus sequences. When people monitor a visual stream for two targets (T1 and T2), they often miss $\mathrm{T} 2$ if it falls into a time window of about 100 $600 \mathrm{msec}$ after T1 onset (Broadbent \& Broadbent, 1987; Raymond, Shapiro, \& Arnell, 1992). In analogy to an overt blink of the eyes, Raymond et al. (1992) have termed this insensitivity to the second of two sequential targets an attentional blink (AB).

Available accounts of the $\mathrm{AB}$ have linked the effect to short-term memory (STM). Consolidation models assume that to report a target, its sensory representation needs to be consolidated into STM, which requires the allocation of attentional resources (Chun \& Potter, 1995; Jolicœur, Dell'Acqua, \& Crebolder, 2000). If resources are allocated to the consolidating of T1 - to a degree and duration that depends on how severely $\mathrm{T} 1$ is masked by subsequent items-fewer resources are left to consolidate T2. This makes T2 codes vulnerable to inhibition from other items competing for access to STM, so that it is less likely to be maintained and reported later on. From a slightly different perspective, interference models assume that it is not the transfer of sensory codes to STM that provides the bottleneck but, rather, the competition between candidate items within STM for being selected for action control (e.g., Duncan, Ward, \& Shapiro, 1994;

This research was supported by a grant from the Volkswagen Foundation. The authors thank Jane Raymond, Kimron Shapiro, and an anonymous reviewer for their comments and helpful suggestions on the way. Address correspondence to E. G. Akyürek or B. Hommel, Department of Psychology, Cognitive Psychology Unit, Leiden University, Postbus 9555, 2300RB Leiden, The Netherlands (e-mail: akyurek@, fsw.leidenuniv.nl or hommel@fsw.leidenuniv.nl).
Shapiro \& Raymond, 1994). Items are thought to be encoded in STM if they match the template representing the current selection goal, where they receive selection values reflecting that degree. The item with the highest value is then selected for action control, such as verbal report. Since T1 will always receive a high value, T2 is likely to lose the competition for selection against $\mathrm{T} 1$ and/or distractor items that erroneously received high selection values by virtue of appearing briefly before or after $\mathrm{T} 1$ - at least if $\mathrm{T} 2$ appears before the selection of $\mathrm{T} 1$ has been completed.

In view of the strong emphasis available models place on STM, we asked in the present study whether RSVP performance and the $\mathrm{AB}$ in particular would be affected by the content and load of STM induced by a concurrent task. Accordingly, we embedded standard RSVP trials into an STM task in which we had participants retain varying numbers of items. Moreover, since interference models assume that competition within STM is modulated by similarity (with more similar items being thought to compete more strongly; cf. Shapiro \& Raymond, 1994; Shapiro, Raymond, \& Arnell, 1995), we loaded STM with various types of items: items that were taken from the same category as either the targets or the distractors of the RSVP task or items that were unrelated to that task.

From a consolidation point of view, one might speculate that consolidating sensory traces into STM gets more difficult, or takes longer, the more filled-up STM already is. This would be expected to decrease performance overall but to affect the $\mathrm{AB}$ (i.e., the performance drop at lags of 100-600 msec) in particular, because the greater the number of attentional resources allocated to $\mathrm{T} 1$ processing, the fewer there are that are available to consolidate $\mathrm{T} 2$ before it decays. In contrast, the way STM is filled up (i.e., which kind of items STM contains) should not play a major role, so that an impact of 
STM item type would not be expected. Interference models assume that competition increases with the number of items in STM, suggesting again that performance in general-and that around lags of 100-600 msec, in particular-decreases with an increasing number of items in the STM task. Moreover, given their reliance on similarity, interference models strongly suggest that such decrements vary with the similarity between the RSVP target set and the item set of the STM task. If so, load effects should be more pronounced if STM items match the category of T1 and T2 in the RSVP task. By contrast, finding no effect of increasing secondary task difficulty would point to a multiple-channel processing mechanism (Awh et al., 2004).

\section{EXPERIMENT 1}

In Experiment 1, we asked participants to identify and report two digits ( $\mathrm{T} 1$ and $\mathrm{T} 2$ ) presented within a stream of letter distractors. Before each RSVP stream, a memory set containing two, four, or six items was presented, and this set was probed after presentation of the RSVP stream. In different conditions, the set comprised (1) symbols that were unrelated to the AB task, (2) letters (i.e., items from the same category as the RSVP distractors, although set members never matched any actual distractor on a given trial), and (3) digits (i.e., items from the same category as the targets in the AB task, although, again, set members never matched any actual target on a given trial). These conditions allowed us to assess the effects on absolute STM load and the content of that load separately.

\section{Method}

Design. Experimental and analytical variables of the $4 \times 3 \times 3$ mixed factorial design were T2 lag (one, three, five, or eight), STM load (two, four, or six items), and STM content (neutral, distractor related, or target related). Lag and load was varied within subjects, and content was varied between subjects. Dependent measures were accuracy on the STM task, accuracy on T1, and conditional accuracy on $\mathrm{T} 2$ (T2|T1).

Participants. A total of 90 students participated for pay, 30 in each STM content group. They reported having normal or corrected-tonormal vision and were unaware of the purpose of the experiment.

Apparatus and Procedure. The participants were seated behind a standard PC in a small, dimly lit cubicle. Stimuli were presented using the E-Prime experimental software package on a 17 in. monitor, refreshing at $85 \mathrm{~Hz}$. Viewing distance was not strictly fixed but amounted to about $50 \mathrm{~cm}$. Each participant completed 288 experimental and 32 practice trials, which took about $1 \mathrm{~h}$. Instructions emphasized performing both the STM and the RSVP tasks as accurately as possible.

Trials were self-paced and began with the presentation of two, four, or six STM items for 1,000 msec. Neutral STM items were taken from a set of symbol characters (!, @, \#, \$, \%, ^, \&, and *), distractor-related STM items were chosen from a random set of uppercase letters, and target-related STM items were randomly chosen digits. In order to equate set sizes to the eight-symbol pool used in the neutral condition, the other two sets were constructed in a similar fashion. STM letters were randomly selected from the 8 letters left after filling the RSVP stream (26 letters in total, minus 18 for the RSVP task). The set of possible STM items in a trial con- sisted of the six digits that remained after selecting the two RSVP targets (eight in total, minus eight) plus the two digits not used in the RSVP task, 0 and 5. Due to this procedure, STM items never appeared in the RSVP task in the same trial. The current STM set was presented in a row centered on the screen.

After a delay of 2,000 msec, a fixation mark (+) appeared for $200 \mathrm{msec}$ in the center of the screen, followed by the RSVP stream. The stream consisted of two targets and 18 distractors. Each item appeared for approximately $59 \mathrm{msec}$, followed by a $35-\mathrm{msec}$ blank (five and three screen refreshes, respectively). T1 appeared as the seventh, eighth, or ninth item of the stream, randomly chosen. T2 appeared with a lag of one (i.e., as the next item), three, five, or eight items. Lag 8 was specifically chosen so as to fall outside the critical AB interval of about half a second, so that performance on this lag can be taken to represent baseline level. ${ }^{1}$ Target items were always digits (1-9, excluding 5), and distractor items were always capital letters. Items were randomized, except that they never appeared twice in the same trial. A further constraint was that response category (being even or odd) was evenly distributed across trials. Both STM and RSVP items were presented in 16-point Times New Roman font in black (RGB 0, 0, 0) on a gray (RGB 128, 128, 128) background.

After the offset of the RSVP stream and a 1,000-msec blank interval, a single item was presented for $1,000 \mathrm{msec}$ to assess STM performance. Depending on the content group, this was a symbol, a letter, or a digit that had a $50 \%$ probability of being part of the STM set for that trial. After another 500-msec delay, a response screen appeared, prompting an unspeeded yes (was part of the set) or no (was not part) decision by pressing the "J" or the "N" key on the computer keyboard. Then the participants were to indicate at leisure whether T1 and T2 were even or odd by using the "E" and "O" keys. Thus, chance level for the STM, task as well as for T1 and $\mathrm{T} 2$, was $50 \%$. The instructions of the experiment stressed the importance of accuracy on each dependent variable and explicitly discouraged strategic response modes focusing on a specific part of the task.

\section{Results and Discussion}

A significance level of $p<.05$ was adopted for all analyses, Greenhouse-Geisser adjusted wherever appropriate. First, we analyzed performance in the STM task. Accuracy varied with T2 lag $\left[F(2.8,240.1)=3.17, M S_{\mathrm{e}}=\right.$ $0.0041, p<.05]$, STM load $[F(1.9,162)=168.89$, $\left.M S_{\mathrm{e}}=0.0080, p<.001\right]$, and STM content $[F(2,87)=$ 22.95, $\left.M S_{\mathrm{e}}=0.0547, p<.001\right]$. Load and content were also involved in a two-way interaction $[F(2,174)=$ 40.02, $\left.M S_{\mathrm{e}}=0.0075, p<.001\right]$.

The lag effect reflected a slight drop in performance if T2 appeared with the longest lag $(88.6 \%, 88.4 \%, 89.3 \%$, and $87.7 \%$ for lags $1,3,5$, and 8 ), presumably due to the fact that the longest lag between T1 and T2 implies the shortest interval between T2 storage and STM test - that is, the shortest time to consolidate T2. Importantly, however, performance for two STM items was very good and about the same in all three groups, suggesting that the participants were motivated and comparable. ${ }^{2}$ The interaction between load and content indicated that in correspondence with the purpose of the load manipulation, accuracy decreased with an increasing number of items, but this decrease was more dramatic with abstract symbols, as can be seen from Table 1 .

Performance on $\mathrm{T} 1$ depended on lag $[F(2.3,201.5)=$ $\left.119.09, M S_{\mathrm{e}}=0.012, p<.001\right], \operatorname{load}[F(2,174)=7.79$, 
Table 1

Mean Short-Term Memory (STM) Performance in Experiment 1 in Percentage Correct as a Function of STM Content, STM Load (Number of Items), and Temporal Lag Between T1 and T2

\begin{tabular}{lccccc}
\hline & & \multicolumn{4}{c}{ Lag } \\
\cline { 3 - 6 } Content & Load & 1 & 3 & 5 & 8 \\
\hline Symbols & 2 & 93.5 & 93.6 & 94.9 & 93.1 \\
& 4 & 79.2 & 81.1 & 81.0 & 81.0 \\
\multirow{3}{*}{ Letters } & 6 & 72.8 & 69.9 & 72.4 & 68.1 \\
& 2 & 94.3 & 93.6 & 95.3 & 92.8 \\
& 4 & 94.3 & 92.9 & 93.3 & 92.1 \\
Digits & 6 & 86.3 & 86.9 & 89.3 & 88.8 \\
& 2 & 94.4 & 95.7 & 94.4 & 94.2 \\
& 4 & 94.4 & 93.8 & 93.3 & 92.1 \\
& 6 & 87.9 & 88.1 & 90.1 & 87.5 \\
\hline
\end{tabular}

$\left.M S_{\mathrm{e}}=0.0071, p<.001\right]$, and content $[F(2,87)=3.60$, $\left.M S_{\mathrm{e}}=0.106, p<.05\right]$. Figure 1 shows mean $\mathrm{T} 1$ response accuracy in all conditions.

The lag effect indicated that performance was particularly poor at Lag 1 , as compared with the other lags. This is a familiar effect - at least in RSVP tasks, where T1 and $\mathrm{T} 2$ are defined according to the same selection criteria (thus enabling direct competition) - that is likely to reflect an attentional tradeoff with T2 (Hommel \& Akyürek, in press; Potter, Staub, \& O'Connor, 2002). The load effect showed that T1 accuracy was worse the greater the number of items that was to be maintained in STM $(83.0 \%, 81.6 \%$, and $80.5 \%$, with two, four, and six STM items, respectively). The content effect was due to better performance when the STM task used neutral symbols than when letters or digits were used.

Our central measure, conditional T2 accuracy, showed a significant effect of $\mathrm{T} 2$ lag $[F(2.6,225.7)=38.82$,
$\left.M S_{\mathrm{e}}=0.0139, p<.001\right]$, reflecting a standard $\mathrm{AB}$ with the typical dip between Lag 1 (Lag 1 sparing; Chun \& Potter, 1995; Potter, Chun, Banks, \& Muckenhoupt, 1998; Potter et al., 2002) and longer lags (see Figure 2). The lag effect was small, presumably due to the rather high $50 \%$ chance level, but robust; for example, it survived dropping a random $50 \%$ of the participants $[F(3,132)=$ $\left.7.17, M S_{\mathrm{e}}=0.0072, p<.001\right]$. Further main effects were obtained for STM load $\left[F(2,174)=10.17, M S_{\mathrm{e}}=0.0056\right.$, $p<.001]$ and STM content $\left[F(2,87)=4.17, M S_{\mathrm{e}}=\right.$ $0.113, p<.05]$, both of which were involved in an interaction that was marginally significant $[F(4,174)=2.35$, $\left.M S_{\mathrm{e}}=0.0056, p<.06\right]$. As Figure 2 shows, performance on T2 decreased with increasing STM load $[86.4 \%, 85.5 \%$, and $83.9 \%$, for two, four, and six items, a linear trend; $F(1,87)=20.42, M S_{\mathrm{e}}=0.0055, p<$ $.001]$, and this effect tended to be most pronounced with the target-related STM set. T2 performance was also better with neutral STM items $(89.4 \%)$ than with distractoror target-related items $(83.4 \%$ and $82.9 \%)$. Of particular interest for our purposes, there was no evidence that any of the effects above or other effects depended on lag (all $\left.F_{\text {s }}<1\right)$. To be certain that there were no isolated interactions of lag and load within the content groups, we looked at these separately and found no significant interactions $(p>.24)$. We also checked whether the opposite roles of neutral items (impairing STM performance but facilitating $\mathrm{T} 2$ report) might indicate a tradeoff. This can be ruled out, however, since the correlations between overall performance in the two tasks were positive in all three content groups (for symbols, $r^{2}=.42, p<.05$; for letters, $r^{2}=.54, p<.001$; for digits, $r^{2}=.40, p<.05$ ). Furthermore, content affected $\mathrm{T} 2$ report significantly even in the two-item condition $\left[F(2,87)=3.48, M S_{\mathrm{e}}=\right.$

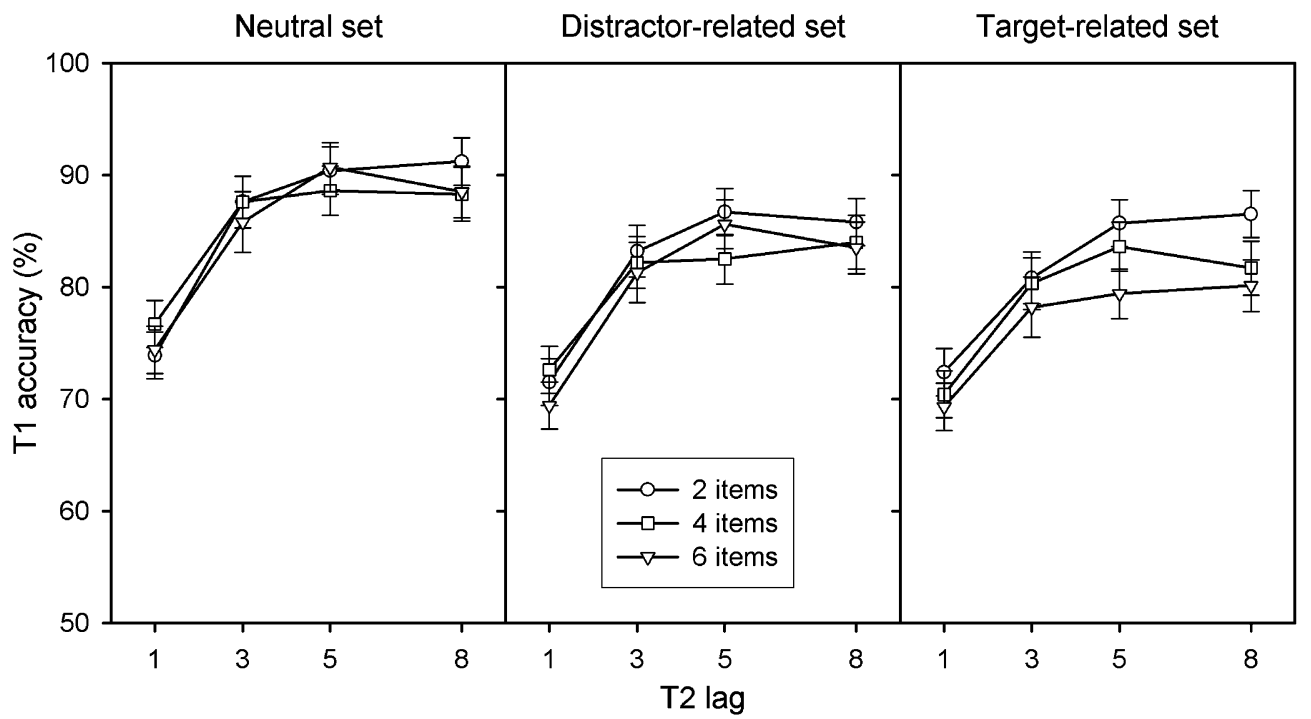

Figure 1. Experiment 1: percentages correct $( \pm 1 S E)$ for the first target, as a function of short-term memory (STM) content, STM load, and T2 lag. 


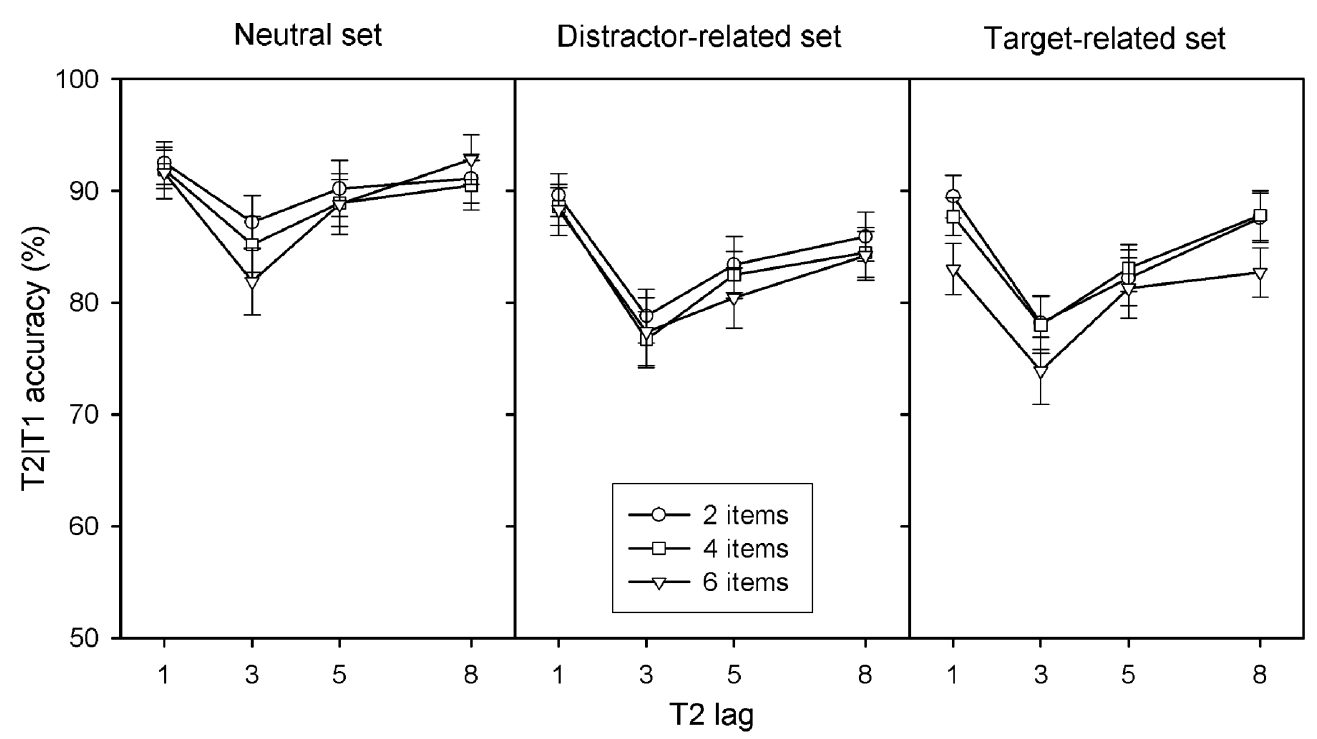

Figure 2. Experiment 1: percentages of correct $( \pm 1 S E)$ conditional classifications of the second target, given the first target (T2|T1), as a function of short-term (STM) content, STM load, and T2 lag.

$0.0393, p<.05]$, in which STM performance was the same for all contents $[F(2,87)<1]$.

To summarize, Experiment 1 produced three results of theoretical relevance: (1) Performance on T2 decreased with increasing memory load and (2) did so depending on the task relevance of the memory set, but (3) neither of these effects interacted with lag.

Interference models have assumed that the $\mathrm{AB}$ reflects interference in STM (Duncan et al., 1994; Shapiro \& Raymond, 1994), which, in the present context, suggested two predictions. First, performance on T2 should decrease under conditions that are likely to increase competition in STM. Experiment 1 showed performance to be impaired as STM load increased and/or as STM content was more task relevant and, thus, was more easily confused with targets in the RSVP task. However, a second, related prediction was that the degree of competition in STM should be particularly important for the time interval following T1 presentation. For instance, Shapiro and Raymond (1994) assumed that T2 processing is affected by competition with other elements in STM for only about half a second from T1 appearance on. Statistically, this amounts to an interaction of competition-inducing variables (here, STM content and load) with lag, and this is an effect that we did not observe in Experiment 1. This might indicate that interference models are correct to assume that competition in STM affects T2 processing but may be insufficient to account for the drop of T2 performance at short lags - hence, the AB proper. However, since this conclusion was based on a null effect - that is, on the absence of an interaction-we sought converging evidence in two additional experiments.

In Experiment 1, we used alphanumeric stimulus materials in both the RSVP and the STM tasks - that is, ma- terial that could be coded visually as well as verbally. This might have introduced the possibility for participants to code STM and RSVP items in different ways, thus eliminating crosstalk. In a way, the fact that we obtained main effects of STM content and STM load speaks against the possibility that the participants had coded the stimuli differently in the two tasks (e.g., verbally in the STM task and visually in the RSVP task), which would have eliminated mutual interference-for example, by running the STM task in the phonological loop (Baddeley, 1986) and the RSVP task in visual STM (Logie, 1995). This means that the absence of a load $X$ lag interaction is unlikely to have been the result of differential-coding strategies. Moreover, if that were to have happened, one would expect less interference for the easier-to-name letters and digits than for the symbols. If anything, however, RSVP performance was affected more by letters and, in particular, by digits as STM items than it was by symbols. Thus, the two tasks must have shared some sort of processing resources (as the consolidation approach suggests) and/or have suffered from some sort of direct crosstalk (as the interference approach suggests). And yet, we thought it would strengthen the case against the interference account of $A B$ if we were able to replicate the null interaction between lag and load under conditions that minimized the opportunity to code STM items and RSVP targets differently.

\section{EXPERIMENT 2}

In Experiment 2, we employed arbitrary, meaningless visual symbols that were unlikely to invite verbal coding. Previous studies have shown that substantial ABs can be obtained with nonverbal material, such as sym- 


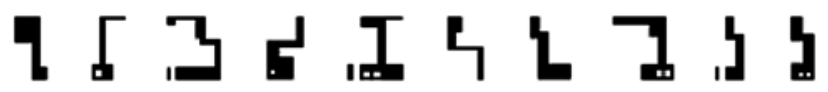

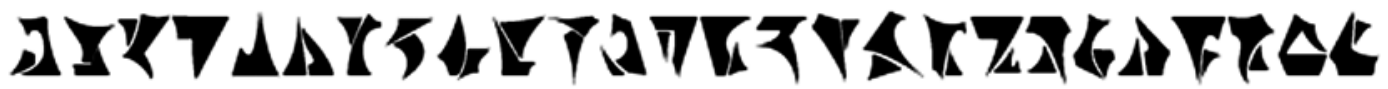

\begin{abstract}
Figure 3. Experiment 2: complete symbol set used. The short-term memory/rapid serial visual respresentation (RSVP) target set is on top, and the RSVP distractor set is below.
\end{abstract}

bols (<, >,\#,\%, ?, /, and *; Chun \& Potter, 1995), visual patterns (Kellie \& Shapiro, 2004), meaningless visual shapes (Chun \& Jiang, 1999; Raymond, 2003), colors (Ross \& Jolicœur, 1999), and time intervals (Sheppard, Duncan, Shapiro, \& Hillstrom, 2002). Here we used "letters" from two "Star Trek" alphabets (see Figure 3). To the degree that the absence of load $\times$ lag interactions in Experiment 1 was due to differential coding (verbal vs. visual) in the RSVP and the STM task, preventing differential coding in Experiment 2 by eliminating the verbal option should yield a substantial interaction.

\section{Method}

Another 46 students participated for pay or course credit. The method and procedure were the same as those in Experiment 1, with the following exceptions. The STM content variable was dropped; only target-relevant STM items were used. STM load was reduced to one, two, or three items, because pilot runs indicated that the new stimuli made the task much more difficult — so difficult that performance often dropped to near-chance level with the original set sizes of two, four, and six items. The visual symbols serving as both STM items and targets for the RSVP stream consisted of a set of 10 letters from the "Cardassian alphabet" used in the fictional "Star Trek" television series (taken from http://www.voyager.fsworld. co.uk/voyfont.htm). These symbols were chosen because they have no apparent meaning, yet do offer a letterlike appearance and a suitable variety at the same time. As in Experiment 1, a symbol never appeared in both the STM and the RSVP tasks on any given trial. The distractors of the RSVP stream were selected from a set of 26 letters from the "Klingon" font of the Star Trek series. Both complete symbol sets are shown in Figure 3.

The intertask interval was eliminated to save session time, a modification that, according to pilot testing, did not affect task performance. ${ }^{3}$ Thus, the RSVP stream started off immediately after the STM set had been presented. Each symbol in the stream was presented for $75 \mathrm{msec}$, followed by a $50 \mathrm{msec}$ blank. T2 appeared with a lag of one, two, three, four, five, or eight items. The background color was changed from gray to white, to increase the discriminability of the stimuli. Font size was set to 24 points, with spacing proportional to that in Experiment 1, to ease identification of the symbols. After the offset of the last RSVP symbol, a 250-msec blank interval ensued, followed by the presentation of the STM probe for $1,000 \mathrm{msec}$. After another $250 \mathrm{msec}$, the participants were prompted to judge whether the probe was part of the STM set. Then the participants were asked to identify the RSVP targets by pressing the corresponding keys on a relabeled keyboard. As a result of changes in the tested conditions, the total number of trials for each participant was 232, 16 of which were practice trials and were not included in the analyses.

\section{Results and Discussion}

Performance on the STM task showed a main effect of load $\left[F(1.7,75)=219.03, M S_{\mathrm{e}}=0.0242, p<.001\right]$, similar to that found in Experiment 1: Accuracy was best with one item, followed by two and three items. The interaction of lag and load also proved to be significant $\left[F(10,450)=2.20, M S_{\mathrm{e}}=0.0129, p<.05\right]$. Although load tended to have a linear impact on performance, the twoitem load condition showed some fluctuation. Performance on Lags 1, 3, and 5 was slightly better than that on Lags 2, 4 , and 8. The relevant means are shown in Table 2.

$\mathrm{T} 1$ and $\mathrm{T} 2$ responses were scored as correct whenever the identity of the respective target was retained, independently of the order of report - a procedure that has the advantage of being consistent with common scoring practice in $\mathrm{AB}$ research but the disadvantage of ignoring the possible loss of order information (Hommel \& Akyürek, in press). Note that this method of analysis is not meaningful for binary category judgments, such as those used in Experiment 1, because identical responses cannot be ordered. Since Experiments 2 and 3 (see below) did require full target identification, the same method was used for both. In addition, we ran control analyses with identity and order as accuracy criteria and found the pattern of outcomes more or less unchanged, especially with regard to the crucial interactions involving load and lag.

T1 performance (see Figure 4) was affected by main effects of T2 lag $\left[F(5,225)=17.63, M S_{\mathrm{e}}=0.021, p<\right.$ $.001]$ and STM load $\left[F(2,90)=10.81, M S_{\mathrm{e}}=0.028\right.$, $p<.001]$. The lag effect reflected a drop of performance on T1 at Lag 1, just as in Experiment 1 (cf. Hommel \& Akyürek, in press; Potter et al., 2002). The load effect indicated better performance when one item $(64.3 \%)$, rather than two $(58.2 \%)$ or three $(59.1 \%)$ items, was to be retained. The difference between conditions here was not very large but may still suggest that more effort is needed to identify a target when the STM load is more than a single item.

Our crucial measure was again T2 performance, contingent on T1 (see Figure 5). As was expected, lag had a

Table 2

Mean Short-Term Memory (STM) Performance in Experiment 2 in Percentage Correct as a Function of STM Load (Number of Items) and Temporal Lag Between T1 and T2

\begin{tabular}{ccccccc}
\hline & \multicolumn{7}{c}{ Lag } \\
\cline { 2 - 7 } Load & 1 & 2 & 3 & 4 & 5 & 8 \\
\hline 1 & 89.1 & 89.7 & 88.0 & 89.9 & 91.3 & 88.4 \\
2 & 78.8 & 71.4 & 78.1 & 72.6 & 78.8 & 71.6 \\
3 & 62.1 & 64.9 & 63.9 & 66.1 & 64.1 & 63.8 \\
\hline
\end{tabular}




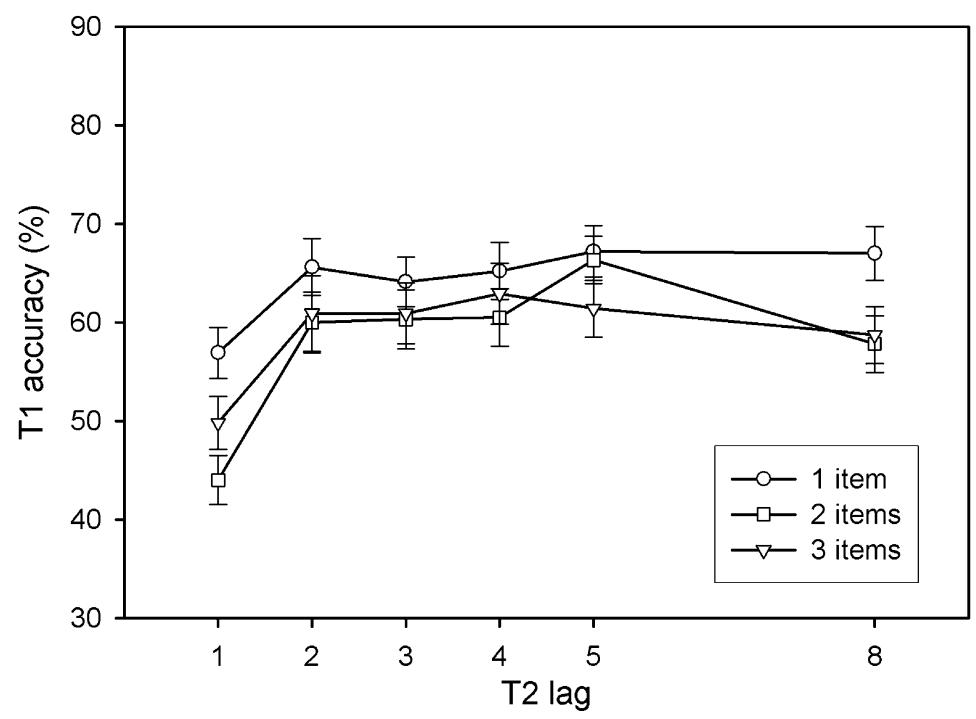

Figure 4. Experiment 2: T1 performance as a function of T2 lag; separate lines represent different short-term memory loads.

significant effect $\left[F(4,179)=35.97, M S_{\mathrm{e}}=0.053, p<\right.$ $.001]$, which represented a typical $\mathrm{AB}$, with performance dipping as low as $33.1 \%$ at Lag 2 and recovering to $62.1 \%$ at Lag 8. There was also a hint of Lag 1 sparing, but it was a modest difference at best. A possible explanation for this small sparing effect may be the fact that both target discrimination (from the stream) and identification must have been much more difficult than in a usual RSVP task, which again is likely to motivate the investment of more attentional resources in $\mathrm{T} 1$ processing-leaving fewer for $\mathrm{T} 2$ to take advantage of the close temporal distance. T2 performance also decreased with increasing
STM load $[48.6 \%, 45.0 \%$, and $44.7 \% ; F(2,90)=3.77$, $\left.M S_{\mathrm{e}}=0.034, p<.05\right]$ - a linear trend $[F(1,45)=5.87$, $\left.M S_{\mathrm{e}}=0.035, p<.05\right]$. The most important outcome is, however, that the interaction between lag and load was far from significance $(p>.58)$, and even the qualitative pattern does not suggest that shorter lags will be affected by STM load more than are longer lags. In fact, all three load functions were more or less parallel across lags.

To summarize, Experiment 2 showed that even if both the STM and the RSVP tasks require visual encoding (since there are no preexisting phonological representations for the stimuli that were used), the impact of STM

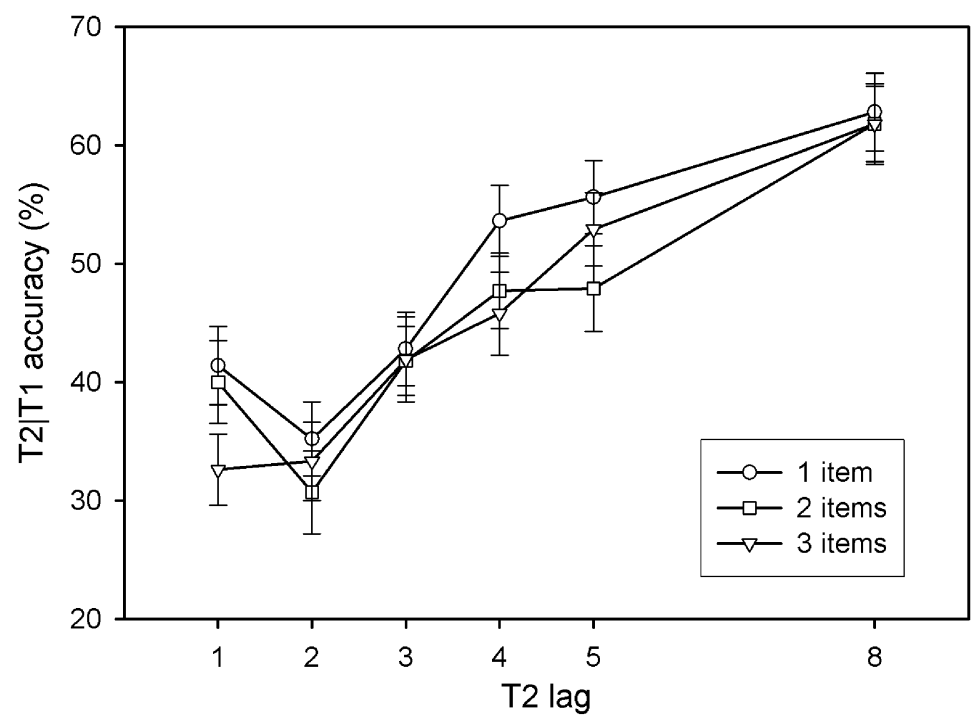

Figure 5. Experiment 2: T2 performance (given T1 correct) as a function of T2 lag; separate lines represent different short-term memory loads. 
load did not increase with decreasing lag. This was true despite the visual task being much more difficult for the participants to perform accurately (therefore leaving more room for error). Thus, Experiment 2 provided further evidence that there is no impact of STM load on the RSVP task that interacts with lag. However, two concerns with Experiment 1 were not addressed: (1) The magnitude of the $\mathrm{AB}$ was rather modest, possibly reducing the chance of finding additional modulation, and (2) the limited impact of increasing load on STM accuracy, which could mean that STM was not fully taxed. In Experiment 3, we attempted to address these two remaining concerns while employing a manipulation similar to the one in Experiment 2 with regard to encoding strategy. This was done by using the same stimulus set as that in Experiment 1 but combining the hybrid RSVP-STM task with a third, verbal suppression task that should prevent verbal coding in both the RSVP and the STM tasks.

\section{EXPERIMENT 3}

\section{Method}

Forty new participants took part in the experiment for pay or course credit: 20 in the suppression group, and 20 in the no-suppression group. The procedure was almost identical to that for the targetrelated condition in Experiment 1, with the addition of the articulatory suppression variable. The differences in procedure included changing the RSVP task to full target identification, instead of a category judgment, and using the intertask intervals from Experiment 2. The participants in the articulatory suppression group were required to repeat the word Maandag (Monday) out loud during each trial.
Table 3

Mean Short-Term Memory (STM) Performance in Experiment 3 in Percentage Correct as a Function of Verbal Suppression, STM Load (Number of Items), and Temporal Lag Between T1 and T2

\begin{tabular}{cccccc}
\hline \multirow{2}{*}{ Suppression } & Load & 1 & 3 & 5 & 8 \\
\cline { 3 - 6 } Yes & 2 & 83.9 & 85.3 & 86.4 & 83.2 \\
& 4 & 69.6 & 70.1 & 69.3 & 66.3 \\
\multirow{3}{*}{ No } & 6 & 60.0 & 59.0 & 59.0 & 53.8 \\
& 2 & 92.5 & 93.5 & 93.1 & 91.1 \\
& 4 & 85.3 & 85.1 & 85.7 & 84.3 \\
& 6 & 74.6 & 74.2 & 74.7 & 74.4 \\
\hline
\end{tabular}

\section{Results and Discussion}

The data were analyzed as a function of T2 lag, STM load, and verbal suppression. STM performance depended on lag $\left[F(3,114)=4.55, M S_{\mathrm{e}}=0.0037, p<.005\right]$ and load $\left[F(1.7,64.9)=115.5, M S_{\mathrm{e}}=0.0203, p<.001\right]$. The lag effect was again limited to a very slight $(\sim 2 \%)$ drop at the longest lag - that is, when the time to consolidate was shortest (means of $77.7 \%, 77.9 \%, 78 \%$, and $75.5 \%$ for the four lags). As was intended, increasing the number of STM items made the task more difficult $(88.6 \%, 77 \%$, and $66.2 \%$ for loads of two, four, and six items, respectively). The suppression variable was also significant $\left[F(1,38)=20.36, M S_{\mathrm{e}}=0.108, p<.001\right]$, as was its interaction with load $\left[F(2,76)=5.62, M S_{\mathrm{e}}=\right.$ $0.0174, p<.005]$. The complete set of means is given in Table 3.

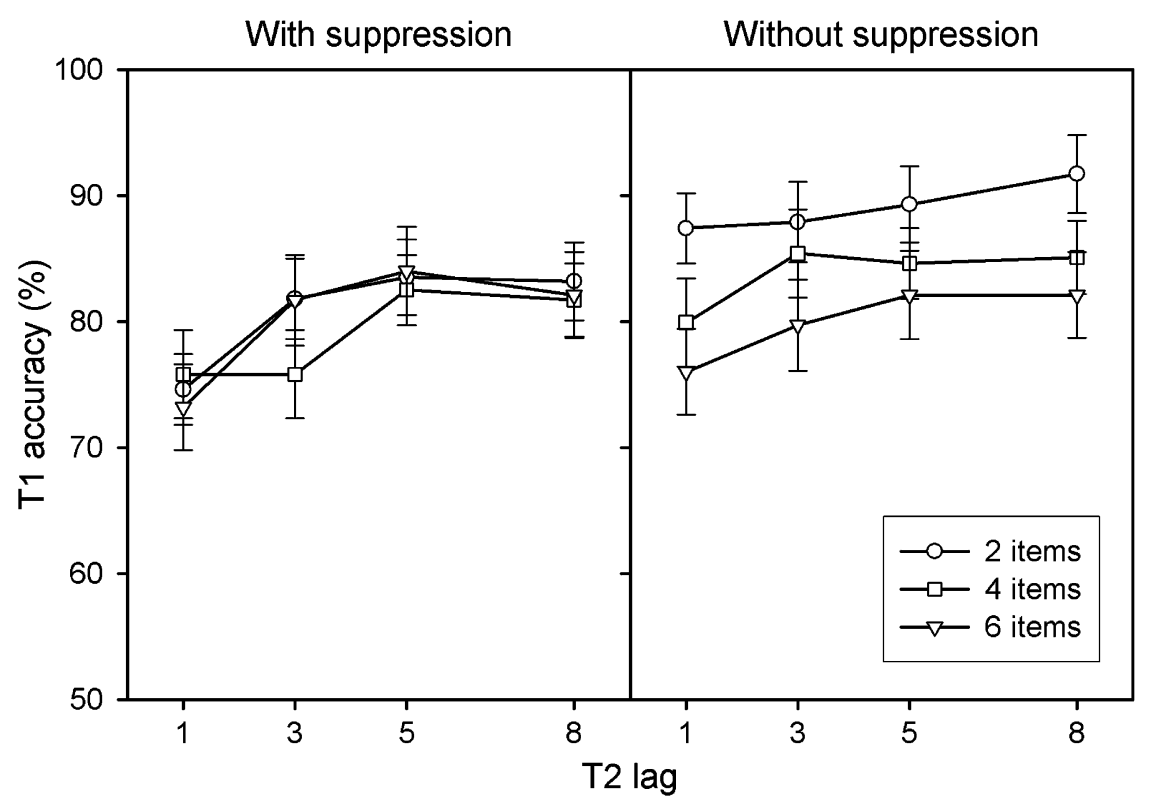

Figure 6. Experiment 3: T1 performance as a function of T2 lag for each short-term memory load. Left panel shows performance with articulatory suppression; right panel shows performance without. 
Unsurprisingly, articulatory suppression added to task difficulty, suggesting an increase of stimulus- and loadindependent task coordination costs (Miyake, Emerson, Padilla, \& Ahn, 2004). The finding that suppression also produced what seems to be a slight increase of the load effect needs to be taken with caution, however: This interaction was entirely due to a modest reduction of the suppression effect with the lowest load, whereas the two conditions with higher loads were equally affected by suppression - a pattern that looked very much like a ceiling effect.

T1 performance varied with lag $[F(3,114)=21.7$, $\left.M S_{\mathrm{e}}=0.005, p<.001\right]$, due to a drop in performance at Lag 1 (see Figure 6). As in Experiment 1, the competition between $\mathrm{T} 1$ and $\mathrm{T} 2$ at this lag was somewhat biased toward $\mathrm{T} 2$ at the expense of $\mathrm{T} 1$ identity, presumably because our targets were again defined according to the same selection criteria. There was also a main effect of load $\left[F(2,76)=8.78, M S_{\mathrm{e}}=0.011, p<.001\right]$, replicating the findings from Experiments 1 and 2. Interestingly, this load effect was modified by suppression $[F(2,76)=$ $\left.6.56, M S_{\mathrm{e}}=0.011, p<.005\right]$. As Figure 6 shows, articulatory suppression reduced the overall performance and leveled out the load effect. Hence, even if the small load effect obtained in Experiments 1 and 2, and in the present no-suppression group, could be taken to point to the use of verbal-coding strategies in the STM task, no such hint remained in the suppression group.

Conditional T2 performance yielded the usual main effect of T2 lag $\left[F(1.8,68.5)=40.7, M S_{\mathrm{e}}=0.04, p<\right.$ $.001]$, showing a standard, AB-type dip at Lag 3 and Lag 1 sparing, shown in Figure 7. The interaction of load and suppression $\left[F(2,76)=2.61, M S_{\mathrm{e}}=0.011, p<.08\right]$ was marginally significant, which reflected a pattern similar to that obtained for T1 performance. Most important, there was no hint of any interaction involving lag $(p>$ .57 ), despite the rapid decline in STM performance observed with increasing load, which suggested that STM capacity was at its limit. Since no main effect of load or suppression was significant, a separate analysis on the no-suppression group was done that showed a main effect of load $\left[F(1.4,27.4)=3.94, M S_{\mathrm{e}}=0.0151, p<\right.$ $.05]$, thus replicating the results in the previous experiments. Mean percentage correct went from $85.8 \%$ to $82.1 \%$ and, finally, to $81.6 \%$ for loads of two, four, and six items, respectively.

As compared with Experiment 1, the concerns regarding AB magnitude and the difficulty of STM load increase were successfully addressed. A blink of sizeable proportion was obtained, and STM performance decreased steadily with increasing load-an indicator of task difficulty. At the same time, the pattern of results remained similar. T2 lag and STM load had effects similar to those observed in the experiments described above, and again, an interaction between them did not show up. One additional observation concerned the reduced impact of STM load on T1 and T2 accuracy in the articulatory suppression condition. Although somewhat mysterious at first glance, this phenomenon could be explained by assuming that a performance floor level was being reached. Suppression caused substantially lower RSVP performance, which in turn may have led to reduced room for additional variance as the participants coded stimuli with reduced but stable efficiency. In sum, Ex-

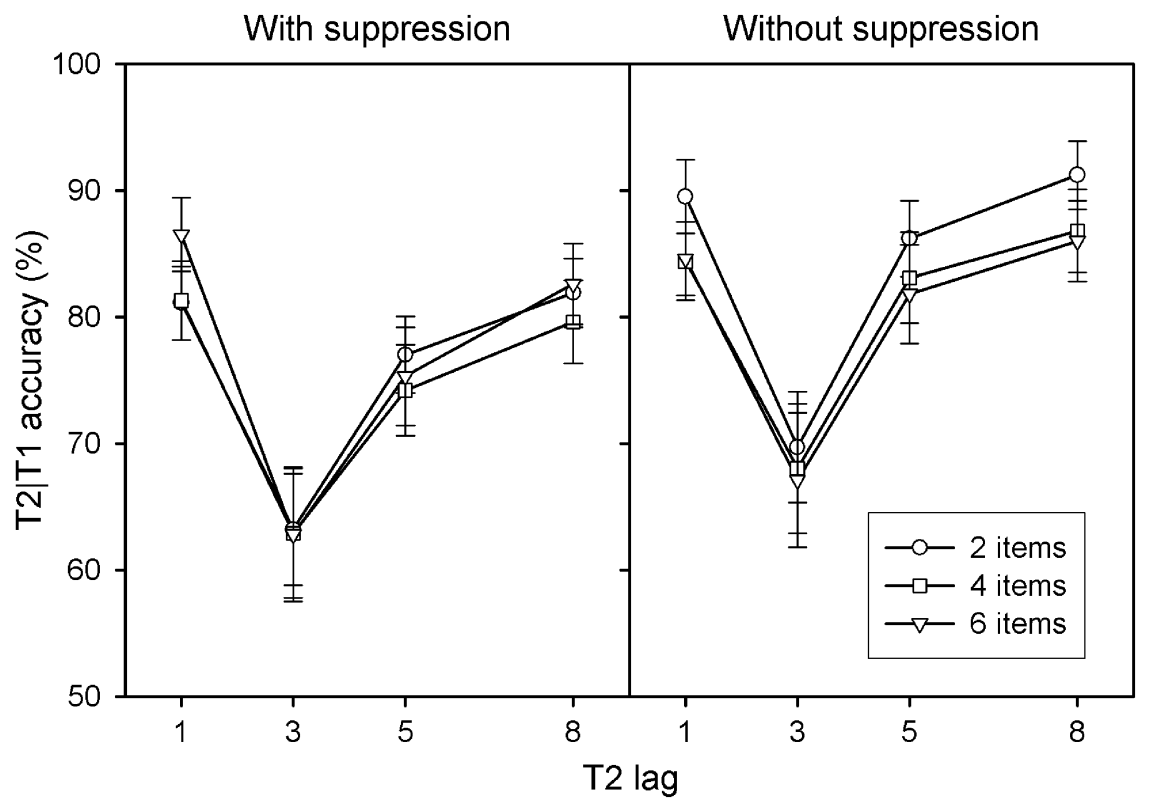

Figure 7. Experiment 3: T2 performance (given $T 1$ correct) as a function of $T 2$ lag for each short-term memory load. Left panel shows performance with articulatory suppression; right panel shows performance without. 
periment 3 provided additional support for the conclusions tentatively drawn in Experiments 1 and 2.

\section{GENERAL DISCUSSION}

Although for different reasons, interference and consolidation accounts of $\mathrm{AB}$ have suggested that performance in an RSVP task is hampered by a concurrent STM task. In the present experiments, STM load impaired both $\mathrm{T} 1$ and $\mathrm{T} 2$ report, and it did so with both alphanumeric stimuli and meaningless symbols. This provided strong evidence that the STM task and the RSVP task shared some sort of processing resources (as the consolidation approach suggests) and/or suffered from some sort of crosstalk (as the interference approach suggests).

A second important observation is that both $\mathrm{T} 1$ and T2 performance were affected by the task relevance of STM items: Memorized items belonging to the same category as RSVP distractors or targets hampered performance more than neutral items did. This is inconsistent with a pure capacity approach, unless one assumes that maintaining overlearned digits and letters requires more capacity than does maintaining abstract symbols. But this assumption received little support from STM performance, which, instead, provided evidence that symbols were the most difficult items. Given that consolidation approaches have not much to say about what goes on after consolidation has taken place, we hesitate to consider the observation of content effects as necessarily incompatible with such an approach. What is clear, however, is that such effects provide ample support for the general assumptions of the interference approach that (1) RSVP performance reflects competition between preselected event codes and (2) the degree of competition depends on similarity between the codes involved (or their match with the template used for preselection). Moreover, the finding that category relations were sufficient to induce competition is consistent with Isaak, Shapiro, and Martin's (1999) claim that what counts most is similarity defined at a conceptual or categorical, but not a purely visual, level-even if our findings did not show that physical similarity has no impact.

Although we hesitate to draw strong conclusions from the marginally significant interaction between content and load on T2 performance in Experiment 1, we do point out that this tendency is also consistent with an interference approach. In most RSVP tasks, both targets and distractors are repeated over and over again, which should lead to a strong priming of their codes above their normal base level. Moreover, since target codes receive both bottom-up activation from the target stimuli presented and top-down activation in order to maintain them for later test, these codes must be particularly primed. Accordingly, items that had served as targets in previous trials and that are expected to appear as targets in later trials should represent particularly strong competitors for selection in STM. If the activation of these codes is further enhanced by making them items in the STM task, it is not surprising to see that they impair T2 report more strongly than others do, and that this impairment is stronger the greater the number of target-related items currently memorized in STM.

With regard to the $\mathrm{AB}$ effect proper, the theoretical implications of our findings are clear as well: We did not find any interaction of either load or content effects with lag, which we take to speak against an interference account of AB. That is, besides any competition within STM, there needs to be some additional capacity bottleneck that excludes the entry of new information while the processing of older information is not yet completedjust as the consolidation account proposes. Obviously, the effects of increasing difficulty within and between tasks show that task overlap is a reality, which speaks against a multiple-channel approach as far as the present paradigm is concerned.

It also seems clear that specific loading of visual STM and phonological subsystems does not change the overall picture, despite indications of increased task difficulty. It is unlikely that either visual STM or a phonological subsystem can account for any substantial part of the $\mathrm{AB}$ deficit. Although it must be kept in mind that, in the case of articulatory suppression, there were several interactions that did show an effect, none of these involved T2 lag.

All in all, our findings provided support for both consolidation and interference models of RSVP performance: Whereas the AB proper seems to be caused by a temporal capacity problem, as consolidation models hold, the overall performance level is considerably influenced by competition from other contents of STM, the more so the more task relevant these competitors are.

\section{REFERENCES}

Awh, E., Serences, J., Laurey, P., Dhaliwal, H., van der Jagt, T., \& DAssonville, P. (2004). Evidence against a central bottleneck during the attentional blink: Multiple channels for configural and featural processing. Cognitive Psychology, 48, 95-126.

BADDELEY, A. D. (1986). Working memory. Oxford: Oxford University Press.

Broadbent, D. E., \& Broadbent, M. H. P. (1987). From detection to identification: Response to multiple targets in rapid serial visual presentation. Perception \& Psychophysics, 42, 105-113.

Chun, M. M., \& JiAng, Y. (1999, November). Task switching and the attentional blink. Paper presented at the 40th Annual Meeting of the Psychonomic Society, Los Angeles.

Chun, M. M., \& Potter, M. C. (1995). A two-stage model for multiple target detection in rapid serial visual presentation. Journal of Experimental Psychology: Human Perception \& Performance, 21, 109127.

Duncan, J., WARD, R., \& SHAPIRO, K. L. (1994). Direct measurement of attentional dwell time in human vision. Nature, 369, 313-315.

Hommel, B., \& AkyüreK, E. G. (in press). The attentional blink and Lag-1 sparing: Benefits and costs of integrating two events into a single episode. Quarterly Journal of Experimental Psychology.

IsAAK, M. I., SHAPIRO, K. L., \& MARTIN, J. (1999). The attentional blink reflects retrieval competition among multiple rapid serial visual presentation items: Tests of the interference model. Journal of Experimental Psychology: Human Perception \& Performance, 25, 1774-1792. 
Jolicceur, P., Dell'AcQua, R., \& Crebolder, J. (2000). Multitasking performance deficits: Forging links between the attentional blink and the psychological refractory period. In S. Monsell \& J. Driver (Eds.), Control of cognitive processes: Attention and performance XVIII (pp. 309-330). Cambridge, MA: MIT Press.

Kellie, F. J., \& ShaPIRo, K. L. (2004). Object file continuity predicts attentional blink magnitude. Perception \& Psychophysics, 66, 692-712.

LogIE, R. H. (1995). Visuo-spatial working memory. Hove, U.K.: Erlbaum.

Miyake, A., Emerson, M. J., Padilla, F., \& Ahn, J.-C. (2004). Inner speech as a retrieval aid for task goals: The effects of cue type and articulatory suppression in the random task cuing paradigm. Acta Psychologica, 115, 123-142.

Potter, M. C., Chun, M. M., Banks, B. S., \& Muckenhoupt, M. (1998). Two attentional deficits in serial target search: The visual attentional blink and an amodal task-switch deficit. Journal of Experimental Psychology: Learning, Memory, \& Cognition, 25, 979-992.

Potter, M. C., Staub, A., \& O'Connor, D. H. (2002). The time course of competition for attention: Attention is initially labile. Journal of Experimental Psychology: Human Perception \& Performance, 28, 1149-1162.

RAYMOND, J. E. (2003). New objects, not new features, trigger the attentional blink. Psychological Science, 14, 54-59.

RAYMOND, J. E., SHAPIRO, K. L., \& ARNell, K. M. (1992). Temporary suppression of visual processing in an RSVP task: An attentional blink? Journal of Experimental Psychology: Human Perception \& Performance, 18, 849-860.

RAYMOND, J. E., Shapiro, K. L., \& Arnell, K. M. (1995). Similarity determines the attentional blink. Journal of Experimental Psychology: Human Perception \& Performance, 21, 653-662.

Ross, N. E., \& JoLICEUU, P. (1999). Attentional blink for color. Journal of Experimental Psychology: Human Perception \& Performance, 25, 1483-1494.

ScHNEIDER, W., \& SHIFFrin, R. M. (1977). Controlled and automatic human information processing: I. Detection, search, and attention. Psychological Review, 84, 1-66.

SHAPIRO, K. L., \& RAYMOND, J. E. (1994). Temporal allocation of visual attention: Inhibition or interference? In D. Dagenbach \& T. H. Carr (Eds.), Inhibitory processes in attention, memory, and language (pp. 151-188). San Diego: Academic Press.

Shapiro, K. L., Raymond, J. E., \& Arnell, K. M. (1997). The attentional blink. Trends in Cognitive Sciences, 1, 291-296.
Sheppard, D. M., Duncan, J., Shapiro, K. L., \& Hillstrom, A. P. (2002). Objects and events in the attentional blink. Psychological Science, 13, 410-415.

Visser, T. A. W., BISChof, W. F., \& Di Lollo, V. (1999). Attentional switching in spatial and non-spatial domains: Evidence from the attentional blink. Psychological Bulletin, 125, 458-469.

\section{NOTES}

1. In keeping with common practice in dual-task and task-switching research, we prefer comparing long and short lags to determine the $\mathrm{AB}$ effect (e.g., Chun \& Potter, 1995; Visser, Bischof, \& Di Lollo, 1999) over comparing single- and dual-target conditions (e.g., Shapiro, Raymond, \& Arnell, 1997), because the latter invites possible confounds associated with pro- and retroactive interference, task switch costs, task coordination overhead, working memory load, and so forth.

2. It may also be taken into account that STM accuracy is not an exclusive measure of (added) task difficulty. Our STM task was similar to the one used by Schneider and Shiffrin (1977), who reported finding little evidence for increased task difficulty as a function of number of items in accuracy, although there was an effect on reaction time. Although we did not employ reaction time measures, this study does lend support to the idea that having more items is indeed more difficult.

3 . Two pilot experiments were run to see whether the methodological changes from Experiment 1 to 2 might have mattered. The first pilot $(N=20)$ was like Experiment 1 (target-related condition), with the following exceptions: The initial intertask interval was done away with and the ending pauses were like those in Experiment 2, and RSVP responses now required an identification, instead of categorization. The results were very similar to those in Experiment 1, with main effects of load and lag but no interaction between the two. Mean T2|T1 performance was $82.4 \%$, $78.4 \%$, and $76.9 \%$ for two, four, and six items, respectively. The second pilot $(N=20)$ was identical to the first, except for the stimulus material, which was replaced by visual symbols such as those used in Experiment 2 . Here, only lag reached significance. Mean performance was $18.1 \%$ for two items, $21.1 \%$ for four items, and $15.7 \%$ for six items (statistically equal, with a chance level of $12.5 \%$ ). Neither experiment produced a qualitatively deviant result (as compared with the experiments reported).

(Manuscript received May 20, 2003; revision accepted for publication August 9, 2004.) 